Document downloaded from:

http://hdl.handle.net/10251/105866

This paper must be cited as:

Cordero Barbero, A.; Torregrosa Sánchez, JR.; Vassileva, MP. (2017). A family of parametric schemes of arbitrary even order for solving nonlinear models. Journal of Mathematical Chemistry. 55(7):1443-1460. doi:10.1007/s10910-016-0723-7

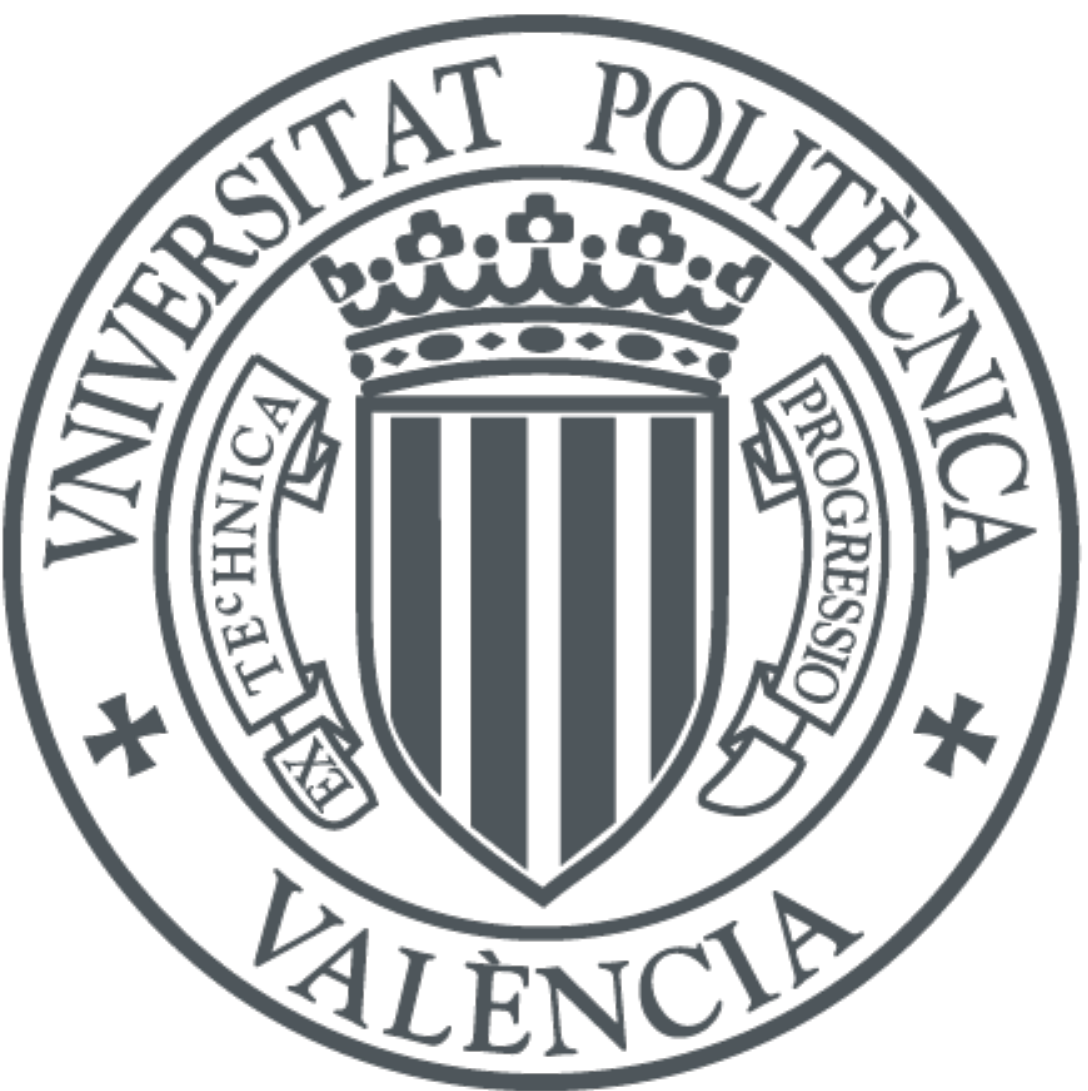

The final publication is available at

http://doi.org/10.1007/s10910-016-0723-7

Copyright Springer-Verlag

Additional Information 


\title{
A family of parametric schemes of arbitrary even order for solving nonlinear models - CMMSE2016
}

\author{
Alicia Cordero · Juan R. Torregrosa • María P. Vassileva
}

Received: date / Accepted: date

\begin{abstract}
Many problems related to gas dynamics, heat transfer or chemical reactions are modeled by means of partial differential equations that usually are solved by using approximation techniques. When they are transformed in nonlinear systems of equations via a discretization process, this system is big-sized and high-order iterative methods are specially useful. In this paper, we construct a new family of parametric iterative methods with arbitrary even order, based on the extension of Ostrowski' and Chun's methods for solving nonlinear systems. Some elements of the proposed class are known methods meanwhile others are new schemes with good properties. Some numerical tests confirm the theoretical results and allow us to compare the numerical results obtained by applying new methods and known ones on academical examples. In addition, we apply one of our methods for approximating the solution of a heat conduction problem described by a parabolic partial differential equation.
\end{abstract}

Keywords System of nonlinear equations - iterative methods · order of convergence $\cdot$ heat conduction problem · divided differences

\section{Introduction}

The solution of nonlinear systems ranks among the most significant classic, frequent and important problem in the theory and practice for many applications in sciences and engineering, see for example the problem of mass and heat transfer within porous catalyst particles, some boundary value problems for integro-differential equations appear that, after accurate discretization, are transformed into a nonlinear system of equations [1,2]. Specifically, we focus our attention to find the solution $\xi$ of a nonlinear system $F(x)=0$, where $F: \Omega \subset \mathbb{R}^{n} \rightarrow \mathbb{R}^{n}$ is a sufficiently Fréchet differentiable function in an open convex set $\Omega$. The best known and used method for solving this kind of problems is Newton's scheme,

$$
x^{(k+1)}=x^{(k)}-\left[F^{\prime}\left(x^{(k)}\right)\right]^{-1} F\left(x^{(k)}\right), k=0,1,2, \ldots,
$$

where $F^{\prime}\left(x^{(k)}\right)$ is the Jacobian matrix of function $F$ evaluated in the $k$ th iteration.

In the literature, one can find multi-step iterative methods for solving systems of nonlinear equations $F(x)=0$ based on Newton's or Newton-like schemes. For example, among other authors, Montazeri et al. in [3] and Hueso et al. in [4], developed sixth-order iterative methods requiring two evaluations of function $F$ and two of Jacobian

This research was partially supported by Ministerio de Economía y Competitividad MTM2014-52016-C02-2-P and FONDOCYT 2014-1C1-088 República Dominicana.

Alicia Cordero

Instituto de Matemática Multidisciplinar, Universitat Politècnica de València,

Camino de Vera, s/n, 46022 Valencia, Spain

E-mail: acordero@mat.upv.es

Juan R. Torregrosa

Instituto de Matemática Multidisciplinar, Universitat Politècnica de València,

Camino de Vera, s/n, 46022 Valencia, Spain

E-mail: jrtorre@mat.upv.es

Maria P. Vassileva

Instituto Tecnológico de Santo Domingo (INTEC),

Santo Domingo, Dominican Republic

E-mail: maria.penkova@intec.edu.do 
$F^{\prime}$ per iteration; moreover, Sharma and Arora in [5] designed a sixth-order method which require three functional and two Jacobian evaluations per iteration. On the other hand, Wang et al. in [6] have constructed a seventh-order derivative-free iterative method by using the first order divided difference operator $[x, y ; F]$ evaluated three times per iteration. The aim of these new schemes is to accelerate the convergence or to improve the computational efficiency.

Among those schemes roughly described previously, we select two of them in order to compare their performance with that of the proposed methods. Sharma et al. in [7] developed several iterative schemes of order six; we use one of them in the numerical section for comparing with our proposed scheme on different test problems. In particular, the authors present the following method that we denote by SA

$$
\begin{aligned}
y^{(k)} & =x^{(k)}-\left[F^{\prime}\left(x^{(k)}\right)\right]^{-1} F\left(x^{(k)}\right), \\
z^{(k)} & =y^{(k)}-\left[3 I-2\left[F^{\prime}\left(x^{(k)}\right)\right]^{-1}\left[x^{(k)}, y^{(k)} ; F\right]\right]\left[F^{\prime}\left(x^{(k)}\right)\right]^{-1} F\left(y^{(k)}\right), \\
x^{(k+1)} & =z^{(k)}-\left[3 I-2\left[F^{\prime}\left(x^{(k)}\right)\right]^{-1}\left[x^{(k)}, y^{(k)} ; F\right]\right]\left[F^{\prime}\left(x^{(k)}\right)\right]^{-1} F\left(z^{(k)}\right) .
\end{aligned}
$$

Wang et al. in [6] describe the following derivative-free seventh-order scheme, that we denote by WZQT

$$
\begin{aligned}
y^{(k)} & =x^{(k)}-B^{-1} F\left(x^{(k)}\right), \\
z^{(k)} & =y^{(k)}-\left[3 I-2 B^{-1}\left[y^{(k)}, x^{(k)} ; F\right]\right] B^{-1} F\left(y^{(k)}\right), \\
x^{(k+1)} & =z^{(k)}-\left[\frac{13}{4} I-B^{-1}\left[z^{(k)}, y^{(k)} ; F\right]\left(\frac{7}{2} I-\frac{5}{4} B^{-1}\left[z^{(k)}, y^{(k)} ; F\right]\right)\right] B^{-1} F\left(z^{(k)}\right),
\end{aligned}
$$

where $B=\left[x^{(k)}+F\left(x^{(k)}\right), x^{(k)}-F\left(x^{(k)}\right) ; F\right]$ and $[\cdot, \cdot ; F]$ is the first order divided difference operator.

In this paper, we design a multidimensional family of bi-parametric iterative schemes (Section 2), whose local order of convergence is stated in Section 3. In Section 4 we present the generalization of the proposed scheme for constructing iterative methods with even arbitrary order of convergence. In order to check the theoretical results, some tests on a heat conduction problem and also on several nonlinear academical systems of equations are made in Section 5. Finally, some conclusions and references are presented.

\section{Design of the methods}

In [8], the authors use a combination of Ostrowski' [9] and Chun's [10] methods for designing a family of fourthorder bi-parametric methods

$$
x_{k+1}=y_{k}-\frac{1}{a}\left[\frac{f\left(x_{k}\right)}{f\left(x_{k}\right)+a(b-2) f\left(y_{k}\right)}+\frac{(a-1) f\left(x_{k}\right)+a b f\left(y_{k}\right)}{f\left(x_{k}\right)}\right] \frac{f\left(y_{k}\right)}{f^{\prime}\left(x_{k}\right)},
$$

where $y_{k}$ is a Newton's step, $y_{k}=x_{k}-\frac{f\left(x_{k}\right)}{f^{\prime}\left(x_{k}\right)}$ and $a$ and $b$ are arbitrary parameters.

Also in [8], some algebraic manipulations allows us to generalize this family to several variables preserving the local order of convergence four by using the divided difference operator $[\cdot, \cdot ; F]: \Omega \times \Omega \subset \mathbb{R}^{n} \times \mathbb{R}^{n} \rightarrow \mathcal{L}\left(\mathbb{R}^{n}\right)$ defined in [12]. Then, the multidimensional version of the class of iterative methods is

$$
\begin{aligned}
x^{(k+1)}= & y^{(k)}-G\left(x^{(k)}, y^{(k)}\right)\left[F^{\prime}\left(x^{(k)}\right)\right]^{-1} F\left(y^{(k)}\right) \\
G\left(x^{(k)}, y^{(k)}\right)= & \frac{1}{a}\left[(1+a b-2 a) I-a(b-2)\left[F^{\prime}\left(x^{(k)}\right)\right]^{-1}\left[x^{(k)}, y^{(k)} ; F\right]\right]^{-1} \\
& +\frac{1}{a}\left[(a+a b-1) I-a b\left[F^{\prime}\left(x^{(k)}\right)\right]^{-1}\left[x^{(k)}, y^{(k)} ; F\right]\right]
\end{aligned}
$$

where $I$ is the identity matrix and $F^{\prime}$ is the Jacobian matrix associated with the nonlinear function. Let us remark that we can rewrite function $G\left(x^{(k)}, y^{(k)}\right)$ as follows

$$
G\left(x^{(k)}, y^{(k)}\right)=\left[r_{1} I+r_{2} S^{(k)}\right]^{-1}\left[k_{1} I+k_{2} S^{(k)}+k_{3}\left[S^{(k)}\right]^{2}\right]
$$

where $k_{1}=3-a b-2 a+a b^{2}, k_{2}=-2-2 a b^{2}+2 a+3 a b, k_{3}=a b(b-2), r_{1}=(1+a b-2 a), r_{2}=-a(b-2)$ and $S^{(k)}=\left[F^{\prime}\left(x^{(k)}\right)\right]^{-1}\left[x^{(k)}, y^{(k)} ; F\right]$. 
If we take a particular case of this family of bi-parametric iterative methods, specifically $b=0$ and any nonzero values of $a$, we obtain

$$
G\left(x^{(k)}, y^{(k)}\right)=\left[(1-2 a) I+2 a S^{(k)}\right]^{-1}\left[(3-2 a) I-2(1-a) S^{(k)}\right] .
$$

Denoting $2 a=2-\beta$, we obtain

$$
G\left(x^{(k)}, y^{(k)}\right)=\left[(\beta-1) I-(\beta-2) S^{(k)}\right]^{-1}\left[(\beta+1) I-\beta S^{(k)}\right],
$$

that is, the multidimensional extension of scalar King's family. Now, if $\beta=2$, the function used in (3) is

$$
G\left(x^{(k)}, y^{(k)}\right)=3 I-2 S^{(k)}
$$

and the resulting scheme is the extension of the Chun's method for systems. However, if $\beta=0$

$$
G\left(x^{(k)}, y^{(k)}\right)=\left[-I+2 S^{(k)}\right]^{-1}
$$

and, replacing $G\left(x^{(k)}, y^{(k)}\right)$ in (3), the extension of the Ostrowski's method for systems is obtained.

In our proposal, the multivariate bi-parametric family $(3)$, where $G\left(x^{(k)}, y^{(k)}\right)$ takes the form (4), is used as a predictor, and we add a corrector step in the following form:

$$
x^{(k+1)}=z^{(k)}-T\left(x^{(k)}, y^{(k)}, z^{(k)}\right)\left[F^{\prime}\left(x^{(k)}\right)\right]^{-1} F\left(z^{(k)}\right),
$$

where $z^{(k)}$ is obtained by using (3) and

$$
T\left(x^{(k)}, y^{(k)}, z^{(k)}\right)=m_{1} I+m_{2} G\left(x^{(k)}, y^{(k)}\right)+m_{3} H\left(y^{(k)}, z^{(k)}\right),
$$

being $G\left(x^{(k)}, y^{(k)}\right)$ defined in (4) and $H\left(y^{(k)}, z^{(k)}\right)$ is calculated as

$$
H\left(y^{(k)}, z^{(k)}\right)=\left(n_{1}+n_{2}\right) I-n_{2} G\left(x^{(k)}, y^{(k)}\right)\left[F^{\prime}\left(x^{(k)}\right)\right]^{-1}\left[y^{(k)}, z^{(k)} ; F\right] .
$$

In what follows, we analyze the values of the introduced parameters in order to assure an order of convergence as higher as possible.

\section{Order of convergence of the new methods}

To show the order of convergence of the new family of bi-parametric iterative schemes, we use the notation defined in [11]. Next, we need to obtain the Taylor expansion of the divided difference operator $[\cdot, \cdot ; F]: \Omega \times \Omega \subset \mathbb{R}^{n} \times \mathbb{R}^{n} \rightarrow$ $\mathcal{L}\left(\mathbb{R}^{n}\right)$ defined in $[12]$, such that $[x, y ; F](x-y)=F(x)-F(y)$, for any $x, y \in \Omega$. For this purpose, we use the Genocchi-Hermite formula (see [13])

$$
[x, x+h ; F]=\int_{0}^{1} F^{\prime}(x+t h) d t
$$

and, by developing $F^{\prime}(x+t h)$ in Taylor series around $x$ (see [8]), we obtain

$$
\int_{0}^{1} F^{\prime}(x+t h) d t=F^{\prime}(x)+\frac{1}{2} F^{\prime \prime}(x) h+\frac{1}{6} F^{\prime \prime \prime}(x) h^{2}+\mathcal{O}\left(h^{3}\right) .
$$

Defining $e=x-\xi$ and assuming that $F^{\prime}(\xi)$ is nonsingular, we have:

$$
\begin{aligned}
F(x) & =F^{\prime}(\xi)\left(e+C_{2} e^{2}+C_{3} e^{3}+C_{4} e^{4}+C_{5} e^{5}+C_{6} e^{6}\right)+\mathcal{O}\left[e^{7}\right], \\
F^{\prime}(x) & =F^{\prime}(\xi)\left(I+2 C_{2} e+3 C_{3} e^{2}+4 C_{4} e^{3}+5 C_{5} e^{4}+6 C_{6} e^{5}\right)+\mathcal{O}\left[e^{6}\right], \\
F^{\prime \prime}(x) & =F^{\prime}(\xi)\left(2 C_{2}+6 C_{3} e+12 C_{4} e^{2}+20 C_{5} e^{3}+30 C_{6} e^{4}\right)+\mathcal{O}\left[e^{5}\right] \\
F^{\prime \prime \prime}(x) & =F^{\prime}(\xi)\left(6 C_{3}+24 C_{4} e+60 C_{5} e^{2}+120 C_{6} e^{3}\right)+\mathcal{O}\left[e^{4}\right]
\end{aligned}
$$


where $I$ denotes the identity matrix and $C_{q}=\frac{1}{q !}\left[F^{\prime}(\xi)\right]^{-1} F^{(q)}(\xi), q \geq 2$. Replacing these developments in the formula of Genocchi-Hermite and denoting the second point of the divide difference by $y=x+h$ and its error respect the solution $\xi$ by $e_{y}=y-\xi$, we have

$$
[x, y ; F]=F^{\prime}(\xi)\left[I+C_{2}\left(e_{y}+e\right)+C_{3} e^{2}\right]+\mathcal{O}\left[e^{3}\right] .
$$

In particular, if $y$ is an approximation to the solution provided by Newton's method, i.e., $h=x-y=\left[F^{\prime}(x)\right]^{-1} F(x)$, we obtain

$$
[x, y ; F]=F^{\prime}(\xi)\left[I+C_{2} e+\left(C_{2}^{2}+C_{3}\right) e^{2}\right]+\mathcal{O}\left[e^{3}\right] .
$$

Theorem 1 Let $F: \Omega \subseteq \mathbb{R}^{n} \rightarrow \mathbb{R}^{n}$ be a sufficiently Fréchet differentiable at $\Omega$ and let $\xi \in \Omega$ be the solution of system of nonlinear equations $F(x)=0$. Let us assume that $F^{\prime}(x)$ is continuous and nonsingular in $\xi$. We consider that $x^{(0)}$ is a initial approximation close enough to $\xi$. Then, sequence $\left\{x^{(k)}\right\}_{k \geq 0}$ obtained by using the iterative scheme

$$
\begin{aligned}
y^{(k)} & =x^{(k)}-\left[F^{\prime}\left(x^{(k)}\right)\right]^{-1} F\left(x^{(k)}\right), \\
z^{(k)} & =y^{(k)}-G\left(x^{(k)}, y^{(k)}\right)\left[F^{\prime}\left(x^{(k)}\right)\right]^{-1} F\left(y^{(k)}\right), \\
x^{(k+1)} & =z^{(k)}-T\left(x^{(k)}, y^{(k)}, z^{(k)}\right)\left[F^{\prime}\left(x^{(k)}\right)\right]^{-1} F\left(z^{(k)}\right),
\end{aligned}
$$

converges to $\xi$ with order of convergence six if $m_{1}=-n_{1} m_{3}$ and $m_{2}=1$ for any $n_{2} \neq 0, n_{1}$ and $m_{3}$. Here, $G\left(x^{(k)}, y^{(k)}\right)$ is according to (4), $T\left(x^{(k)}, y^{(k)}, z^{(k)}\right)$ according to (5) and $H\left(y^{(k)}, z^{(k)}\right)$ according to (6).

Proof : By developing in Taylor series functions $F\left(x^{(k)}\right)$ and $F^{\prime}\left(x^{(k)}\right)$ we obtain

$$
\begin{aligned}
& F\left(x^{(k)}\right)=F^{\prime}(\xi)\left(e_{k}+C_{2} e_{k}^{2}+C_{3} e_{k}^{3}+C_{4} e_{k}^{4}+C_{5} e_{k}^{5}+C_{6} e_{k}^{6}\right)+\mathcal{O}\left[e_{k}^{7}\right], \\
& F^{\prime}\left(x^{(k)}\right)=F^{\prime}(\xi)\left(I+2 C_{2} e_{k}+3 C_{3} e_{k}^{2}+4 C_{4} e_{k}^{3}+5 C_{5} e_{k}^{4}+6 C_{6} e_{k}^{5}\right)+\mathcal{O}\left[e_{k}^{6}\right],
\end{aligned}
$$

where $e_{k}=x^{(k)}-\xi$.

Considering that $\left[F^{\prime}\left(x^{(k)}\right)\right]^{-1} F^{\prime}\left(x^{(k)}\right)=I$, we obtain

$$
\left[F^{\prime}\left(x^{(k)}\right)\right]^{-1}=\left[I+X_{2} e_{k}+X_{3} e_{k}^{2}+X_{4} e_{k}^{3}+X_{5} e_{k}^{4}+X_{6} e_{k}^{5}\right]\left[F^{\prime}(\xi)\right]^{-1}+\mathcal{O}\left[e_{k}^{6}\right]
$$

where $X_{1}=I$ and $X_{m}=-\sum_{j=2}^{m} j X_{m-j+1} C_{j}, m=2,3, \ldots$ So, we calculate the product

$$
\left[F^{\prime}\left(x^{(k)}\right)\right]^{-1} F\left(x^{(k)}\right)=e_{k}+\sum_{j=3}^{s} A_{j} e_{k}^{j}+\mathcal{O}\left[e_{k}^{7}\right]
$$

where $A_{2}=-C_{2}$ and $A_{m}=C_{m}+\sum_{j=3}^{m} X_{m-j+2} C_{j-1}+X_{m}, m=3,4, \ldots$ Thus, $y^{(k)}$ has the form:

$$
y^{(k)}=x^{(k)}-\left[F^{\prime}\left(x^{(k)}\right)\right]^{-1} F\left(x^{(k)}\right)=\xi-A,
$$

where $A=\sum_{j=3}^{6} A_{j} e_{k}^{j}+\mathcal{O}\left[e_{k}^{7}\right]$. Again, by expanding in Taylor series $F\left(y^{(k)}\right)$ and $F^{\prime}\left(y^{(k)}\right)$ :

$$
\begin{aligned}
F\left(y^{(k)}\right) & =F^{\prime}(\xi)\left[B_{2} e_{k}^{2}+B_{3} e_{k}^{3}+B_{4} e_{k}^{4}+B_{5} e_{k}^{5}+B_{6} e_{k}^{6}\right]+\mathcal{O}\left[e_{k}^{7}\right], \\
F^{\prime}\left(y^{(k)}\right) & =F^{\prime}(\xi)\left[I+U_{2} e_{k}^{2}+U_{3} e_{k}^{3}+U_{4} e_{k}^{4}+U_{5} e_{k}^{5}\right]+\mathcal{O}\left[e_{k}^{6}\right],
\end{aligned}
$$

where $B_{2}=-A_{2}, B_{3}=-A_{3}, B_{4}=-A_{4}+C_{2}^{3}, B_{5}=-A_{5}-C_{2}^{2} A_{3}-C_{2} A_{3} C_{2}, B_{6}=-C_{2}^{2} A_{4}+C_{2} A_{3}^{2}-C_{2} A_{4} C_{2}+C_{3} C_{2}^{3}$, $U_{2}=2 C_{2}^{2}, U_{3}=-2 C_{2} A_{3}, U_{4}=3 C_{3} C_{2}^{2}-2 C_{2} A_{4}, U_{5}=-2 C_{2} A_{5}-3 C_{3} C_{2} A_{3}-3 C_{3} A_{3} C_{2}, U_{6}=-2 C_{2} A_{6}-3 C_{3} C_{2} A_{4}+$ $3 C_{3} A_{3}^{2}+3 C_{3} A_{3}^{2}-3 C_{3} A_{4} C_{2}+4 C_{4} C_{2}^{3}$. 
Applying these results, we obtain the Taylor expansion of the divided difference operator $\left[x^{(k)}, y^{(k)} ; F\right]$

$$
\left[x^{(k)}, y^{(k)} ; F\right]=F^{\prime}(\xi)\left(I+F_{1} e_{k}+F_{2} e_{k}^{2}+F_{3} e_{k}^{3}\right)+\mathcal{O}\left[e_{k}^{4}\right],
$$

where $F_{1}=C_{2}, F_{2}=C_{2}^{2}+C_{3}$ and $F_{3}=C_{4}-2 C_{2}^{3}+2 C_{2} C_{3}+C_{3} C_{2}$. Then, we calculate

$$
S=\left[F^{\prime}\left(x^{(k)}\right)\right]^{-1}\left[x^{(k)}, y^{(k)} ; F\right]=I+S_{1} e_{k}+S_{2} e_{k}^{2}+S_{3} e_{k}^{3}+S_{4} e_{k}^{4}+S_{5} e_{k}^{5}+S_{6} e_{k}^{6}+\mathcal{O}\left[e_{k}^{7}\right],
$$

where $S_{1}=F_{1}+X_{2}, S_{m}=F_{m}+\sum_{j=2}^{m} X_{m-j+2} F_{j-1}+X_{m+1}, m=2,3, \ldots$ This allows us to calculate the Taylor development of function $G\left(x^{(k)}, y^{(k)}\right)=\left[M\left(x^{(k)}\right)\right]^{-1} N\left(x^{(k)}\right)$, where

$$
\begin{aligned}
M\left(x^{(k)}\right) & =r_{1} I+r_{2} S=r I+r_{2} S_{1} e_{k}+S_{2} e_{k}^{2}+S_{3} e_{k}^{3}+S_{4} e_{k}^{4}+S_{5} e_{k}^{5}+S_{6} e_{k}^{6}+\mathcal{O}\left[e_{k}^{7}\right], \\
N\left(x^{(k)}\right) & =k_{1} I+k_{2} S+k_{3} S^{2}=k I+N_{1} e_{k}+N_{2} e_{k}^{2}+N_{3} e_{k}^{3}+N_{4} e_{k}^{4}+N_{5} e_{k}^{5}+N_{6} e_{k}^{6}+\mathcal{O}\left[e_{k}^{7}\right],
\end{aligned}
$$

where $k=k_{1}+k_{2}+k_{3}=1, r=r_{1}+r_{2}=1, M_{i}=r_{2} S_{i}, N_{i}=\left(k_{2} S_{i}+k_{3} S_{i}^{\prime}\right), i=1,2, \ldots S_{1}^{\prime}=2 S_{1}, S_{2}^{\prime}=2 S_{2}+S_{1}^{2}$, $S_{3}^{\prime}=2 S_{3}+S_{1} S_{2}+S_{2} S_{1}, S_{4}^{\prime}=2 S_{4}+S_{1} S_{3}+S_{2}^{2}+S_{3} S_{1}, S_{5}^{\prime}=2 S_{5}+S_{1} S_{4}+S_{2} S_{3}+S_{3} S_{2}+S_{4} S_{1}, S_{6}^{\prime}=$ $2 S_{6}+S_{1} S_{5}+S_{2} S_{4}+S_{3}^{2}+S_{4} S_{2}+S_{5} S_{1}$ and we can calculate $\left[M\left(x^{(k)}\right)\right]^{-1}$ recalling that $\left[M\left(x^{(k)}\right)\right]^{-1}\left[M\left(x^{(k)}\right)\right]=I$. So,

$$
\left[M\left(x^{(k)}\right)\right]^{-1}=\left(I+Y_{2} e_{k}+Y_{3} e_{k}^{2}+Y_{4} e_{k}^{3}+Y_{5} e_{k}^{4}+Y_{6} e_{k}^{5}\right)\left[F^{\prime}(\xi)\right]^{-1}+\mathcal{O}\left[e_{k}^{6}\right]
$$

where $Y_{2}=-M_{1}, Y_{m}=-M_{m-1}-\sum_{j=3}^{m} Y_{j-1} M_{m-j+1}, m=2,3, \ldots$ Then,

$$
G\left(x^{(k)}, y^{(k)}\right)=I+G_{1} e_{k}+G_{2} e_{k}^{2}+G_{3} e_{k}^{3}+G_{4} e_{k}^{4}+G_{5} e_{k}^{5}+G_{6} e_{k}^{6}+\mathcal{O}\left[e_{k}^{7}\right],
$$

where $G_{1}=N_{1}+Y_{2}, G_{2}=N_{2}+Y_{2} N_{1}+Y_{3}, G_{m}=N_{m}+\sum_{j=2}^{m} Y_{m-j+2} N_{j-1}+Y_{j+1}, m=2,3, \ldots$ Thus,

$$
\begin{aligned}
W\left(x^{(k)}\right) & =\left[F^{\prime}\left(x^{(k)}\right)\right]^{-1} F\left(y^{(k)}\right)=W_{2} e_{k}^{2}+W_{3} e_{k}^{3}+W_{4} e_{k}^{4}+W_{5} e_{k}^{5}+W_{6} e_{k}^{6}+\mathcal{O}\left[e_{k}^{7}\right], \\
R\left(x^{(k)}\right) & =G\left(x^{(k)}, y^{(k)}\right) W\left(x^{(k)}\right)=R_{2} e_{k}^{2}+R_{3} e_{k}^{3}+R_{4} e_{k}^{4}+R_{5} e_{k}^{5}+R_{6} e_{k}^{6}+\mathcal{O}\left[e_{k}^{7}\right]
\end{aligned}
$$

where $W_{2}=B_{2}, W_{m}=B_{m}+\sum_{j=3}^{m} X_{j-1} B_{m-j+2}, R_{2}=W_{2}$ and $R_{m}=B_{m}+\sum_{j=3}^{m} G_{j-2} W_{m-j+2}$. Finally, we obtain for $z^{(k)}$ the following expression

$$
z^{(k)}=y^{(k)}-R\left(x^{(k)}\right)=\xi+Z_{2} e_{k}^{2}+Z_{3} e_{k}^{3}+Z_{4} e_{k}^{4}+Z_{5} e_{k}^{5}+Z_{6} e_{k}^{6}+\mathcal{O}\left[e_{k}^{7}\right],
$$

where $Z_{i}=-A_{i}-R_{i}, i=2,3, \ldots$. It can be checked that $Z_{2}=Z_{3}=0, Z_{4}=\left\{\left[5-a(b-2)^{2}\right] C_{2}^{2}-C_{3}\right\} C_{2}$. For the next step we need to calculate $F\left(z^{(k)}\right),\left[z^{(k)}, y^{(k)} ; F\right]$ and $T\left(x^{(k)}, y^{(k)}, z^{(k)}\right)$. Using again the Taylor series expansion we obtain the following expression for $F\left(z^{(k)}\right)$,

$$
F\left(z^{(k)}\right)=F^{\prime}(\xi)\left(Z_{4} e_{k}^{4}+Z_{5} e_{k}^{5}+Z_{6} e_{k}^{6}\right)+\mathcal{O}\left[e_{k}^{7}\right],
$$

for $\left[z^{(k)}, y^{(k)} ; F\right]$

$$
\left[z^{(k)}, y^{(k)} ; F\right]=F^{\prime}(\xi)\left(I+P_{2} e_{k}^{2}+P_{3} e_{k}^{3}+P_{4} e_{k}^{4}+P_{5} e_{k}^{5}+P_{6} e_{k}^{6}\right)+\mathcal{O}\left[e_{k}^{7}\right],
$$

where $P_{2}=U_{2}, P_{3}=U_{3}+U_{2} A_{2}$ and $P_{4}=U_{4}+U_{2} A_{3}+\frac{2}{3} U_{3} A_{2}+\frac{1}{3} U_{2} A_{2}^{2}$. From expression of $T\left(x^{(k)}, y^{(k)}, z^{(k)}\right)=$ $\left[m_{1}+\left(n_{1}+n_{2}\right) m_{3}\right] I+m_{2} G\left(x^{(k)}, y^{(k)}\right)-m_{3} n_{2} L\left(x^{(k)}, y^{(k)}, z^{(k)}\right)$ we obtain the following result:

$$
T\left(x^{(k)}, y^{(k)}, z^{(k)}\right)=\left(m_{1}+n_{1} m_{3}+m_{2}\right) I+\sum_{i=1}^{m} T_{i}
$$

where $T_{i}=m_{2} G_{i}-m_{3} n_{2} L_{i}$. We obtain $L_{i}$ multiplying $G\left(x^{(k)}, y^{(k)}\right),\left[F^{\prime}\left(x^{(k)}\right)\right]^{-1}$ and $\left[z^{(k)}, y^{(k)} ; F\right]$. The result of the product of $G\left(x^{(k)}, y^{(k)}\right)$ and $\left[F^{\prime}\left(x^{(k)}\right)\right]^{-1}$ is

$$
Q\left(x^{(k)}\right)=\left[I+Q_{1} e_{k}+Q_{2} e_{k}^{2}+Q_{3} e_{k}^{3}+Q_{4} e_{k}^{4}+Q_{5} e_{k}^{5}+Q_{6} e_{k}^{6}\right]\left[F^{\prime}(\xi)\right]^{-1}+\mathcal{O}\left[e_{k}^{7}\right]
$$


where $Q_{1}=X_{2}+G_{2}$ and $Q_{m}=X_{m}+\sum_{j=2}^{m} G_{j-2} X_{m-j+2}+G_{m-1}$, and the result of multiplying $Q\left(x^{(k)}\right)$ and $\left[z^{(k)}, y^{(k)} ; F\right]$ is

$$
L\left(x^{(k)}\right)=I+L_{1} e_{k}+L_{2} e_{k}^{2}+L_{3} e_{k}^{3}+L_{4} e_{k}^{4}+L_{5} e_{k}^{5}+L_{6} e_{k}^{6}+\mathcal{O}\left[e_{k}^{7}\right]
$$

where $L_{1}=Q_{1}, L_{2}=U_{2}+Q_{2}, L_{m}=P_{m}+\sum_{j=3}^{m} Q_{j-2} P_{m-j+2}+Q_{m}$. The product of $\left[F^{\prime}\left(x^{(k)}\right)\right]^{-1}$ and $F\left(z^{(k)}\right)$ gives

$$
\left[F^{\prime}\left(x^{(k)}\right)\right]^{-1} F\left(z^{(k)}\right)=Z_{4} e_{k}^{4}+\left(Z_{5}+X_{2} Z_{4}\right) e_{k}^{5}+\left(Z_{6}+X_{2} Z_{5}+Z_{6}\right) e_{k}^{6}+\mathcal{O}\left[e_{k}^{7}\right] .
$$

Finally, the expression for $x^{(k+1)}$ is

$$
x^{(k+1)}=z^{(k)}-K\left(x^{(k)}\right)=\xi+(1-t) Z_{4} e_{k}^{4}+\left(Z_{5}-K_{5}\right) e_{k}^{5}+\left(Z_{6}-K_{6}\right) e_{k}^{6}+\mathcal{O}\left[e_{k}^{7}\right]
$$

and the expression of the error equation is

$$
e_{k+1}=(1-t) Z_{4} e_{k}^{4}+\left(Z_{5}-K_{5}\right) e_{k}^{5}+\left(Z_{6}-K_{6}\right) e_{k}^{6}+\mathcal{O}\left[e_{k}^{7}\right]
$$

where $t=m_{1}+m_{2}+n_{1} m_{3}, K_{5}=t\left(Z_{5}+X_{2} Z_{4}\right)+T_{1} Z_{4}$ and $K_{6}=t\left(Z_{6}+X_{2} Z_{5}+Z_{6}\right)+T_{1}\left(Z_{5}+X_{2} Z_{4}\right)+T_{2} Z_{4}$. If $t=1$, then $m_{1}=1-m_{2}-n_{1} m_{3}$, the order of convergence is at least five. In case that $Z_{5}-K_{5}=0$ the order of convergence is at least six. Solving this equation we obtain that this conditions imply $m_{2}=1, m_{1}=-n_{1} m_{3}$ and the expression of the error equation is

$$
e_{k+1}=-\left[\left[-6+5 m_{3} n_{2}+a(b-2)^{2}\left(1-m_{3} n_{2}\right)\right] C_{2}^{2}+\left(1-m_{3} n_{2}\right) C_{3}\right] Z_{4} e_{k}^{6}+\mathcal{O}\left[e_{k}^{7}\right]
$$

and the proof is finished.

By replacing in (5) the conditions on parameters that guarantee the sixth-order of convergence, we obtain the following expression of $T\left(x^{(k)}, y^{(k)}, z^{(k)}\right)$,

$$
T\left(x^{(k)}, y^{(k)}, z^{(k)}\right)=\left(3+n_{2} m_{3}\right) I-2 G\left(x^{(k)}, y^{(k)}\right)-n_{2} m_{3} G\left(x^{(k)}, y^{(k)}\right) R\left(x^{(k)}, y^{(k)}, z^{(k)}\right),
$$

where $R\left(x^{(k)}, y^{(k)}, z^{(k)}\right)=\left[F^{\prime}\left(x^{(k)}\right)\right]^{-1}\left[y^{(k)}, z^{(k)} ; F\right]$. We note that this function contains two divided difference operators, involving a high computational cost. By choosing $m_{3}=0$, we eliminate this problem and $T\left(x^{(k)}, y^{(k)}, z^{(k)}\right)$ takes the form

$$
T\left(x^{(k)}, y^{(k)}, z^{(k)}\right)=3 I-2 S,
$$

that corresponds to the function involving Chun's method at the second step and the 6th-order procedure denoted by SA mentioned in the Introduction.

Another interesting selection of parameters in function $G$, described in (4), is $a=b=1$ and $m_{3}=0$ in function $T$. We denote this scheme by M6, which will be used in the numerical section and whose iterative expression is

$$
\begin{aligned}
y^{(k)} & =x^{(k)}-\left[F^{\prime}\left(x^{(k)}\right)\right]^{-1} F\left(x^{(k)}\right), \\
z^{(k)} & =y^{(k)}-\left(\left[x^{(k)}, y^{(k)} ; F\right]^{-1}+\left[I-\left[F^{\prime}\left(x^{(k)}\right)\right]^{-1}\left[x^{(k)}, y^{(k)} ; F\right]\right]\left[F^{\prime}\left(x^{(k)}\right)\right]^{-1}\right) F\left(y^{(k)}\right), \\
x^{(k+1)} & =z^{(k)}-\left(\left[x^{(k)}, y^{(k)} ; F\right]^{-1}+\left[I-\left[F^{\prime}\left(x^{(k)}\right)\right]^{-1}\left[x^{(k)}, y^{(k)} ; F\right]\right]\left[F^{\prime}\left(x^{(k)}\right)\right]^{-1}\right) F\left(z^{(k)}\right) .
\end{aligned}
$$

\section{Generalization of the proposed family}

Let us consider the three-step iterative family defined by (7) and satisfying the conditions established in Theorem 1. We show that it is enough to add a new step in the form $x^{(k+1)}=v^{(k)}-T\left(x^{(k)}, y^{(k)}, z^{(k)}\right)\left[F^{\prime}\left(x^{k)}\right]^{-1} F\left(v^{(k)}\right)\right.$, where $v^{(k)}$ is the last step of family (7), to increase the order of convergence in two units. This process is generalized in the following result to get arbitrary even order $2 n$ with $n$ steps,

$$
\begin{aligned}
v_{1}{ }^{(k)} & =x^{(k)}-\left[F^{\prime}\left(x^{(k)}\right)\right]^{-1} F\left(x^{(k)}\right), \\
v_{2}{ }^{(k)} & =v_{1}{ }^{(k)}-G\left(x^{(k)}, y^{(k)}\right)\left[F^{\prime}\left(x^{(k)}\right)\right]^{-1} F\left(v_{1}^{(k)}\right), \\
v_{3}{ }^{(k)} & =v_{2}{ }^{(k)}-T\left(x^{(k)}, y^{(k)}, z^{(k)}\right)\left[F^{\prime}\left(x^{(k)}\right)\right]^{-1} F\left(v_{2}{ }^{(k)}\right), \\
& \vdots \\
v_{n}{ }^{(k)} & =v_{n-1}{ }^{(k)}-T\left(x^{(k)}, y^{(k)}, z^{(k)}\right)\left[F^{\prime}\left(x^{(k)}\right)\right]^{-1} F\left(v_{n-1}{ }^{(k)}\right) .
\end{aligned}
$$


Theorem 2 Let $v_{n}{ }^{(k)}$ be the last step of the process (10) with order of convergence $p=2 n$, with $n \geq 3$. By adding a new step in the form $x^{(k+1)}=v_{n}{ }^{(k)}-T\left(x^{(k)}, y^{(k)}, z^{(k)}\right)\left[F^{\prime}\left(x^{(k)}\right)\right]^{-1} F\left(v_{n}{ }^{(k)}\right)$ we obtain a new method which order of convergence is $p+2$.

Proof As $v_{n}{ }^{(k)}$ has order $p$, its Taylor expansion is

$$
v_{n}{ }^{(k)}=\xi+V_{p} e_{k}^{p}+V_{p+1} e_{k}^{p+1}+V_{p+2} e_{k}^{p+2}+\mathcal{O}\left[e_{k}^{p+3}\right] .
$$

Then,

$$
F\left(v_{n}{ }^{(k)}\right)=F^{\prime}(\xi)\left(V_{p} e_{k}^{p}+V_{p+1} e_{k}^{p+1}+V_{p+2} e_{k}^{p+2}\right)+\mathcal{O}\left[e_{k}^{p+3}\right]
$$

and

$$
\begin{aligned}
G\left(x^{(k)}, y^{(k)}\right)\left[F^{\prime}\left(x^{(k)}\right)\right]^{-1} F\left(v_{n}{ }^{(k)}\right)= & V_{p} e_{k}^{p}+\left(V_{p+1}+X_{2} V_{p}+G_{1} V_{p+2}\right) e_{k}^{p+1} \\
& +\left(V_{p+2}+X_{2} V_{p+1}+X_{3} V_{p}+G_{1} V_{p+1}+G_{1} X_{2} V_{p}+G_{2} V_{p}\right) e_{k}^{p+2}+\mathcal{O}\left[e_{k}^{p+3}\right]
\end{aligned}
$$

where $G_{i}, X_{i}, i=2,3, \ldots$ were defined in the proof of Theorem 1 . Therefore,

$$
\begin{aligned}
e_{k+1} & =\left(v_{n}{ }^{(k)}-\xi\right)-G\left(x^{(k)}, y^{(k)}\right)\left[F^{\prime}\left(x^{(k)}\right)\right]^{-1} F\left(v^{(k)}\right) \\
& =-\left(X_{2}+G_{1}\right) V_{p+2} e_{k}^{p+1}-\left[\left(X_{2}+G_{1}\right) V_{p+1}+\left(X_{3}+G_{1} X_{2}+G_{2}\right) V_{p}\right] e_{k}^{p+2}+\mathcal{O}\left[e_{k}^{p+3}\right] .
\end{aligned}
$$

But $X_{2}=-2 C_{2}$ and $G_{1}=2 C_{2}$ and, therefore,

$$
e_{k+1}=-\left(X_{3}+G_{1} X_{2}+G_{2}\right) V_{p} e_{k}^{p+2}+\mathcal{O}\left[e_{k}^{p+3}\right] .
$$

On the other hand, $X_{3}+G_{1} X_{2}+G_{2}=\left[-6+a(b-2)^{2}\right] C_{2}^{2}+C_{3}$. Then,

$$
e_{k+1}=-\left(\left[-6+a(b-2)^{2}\right] C_{2}^{2}+C_{3}\right) V_{p} e_{k}^{p+2}+\mathcal{O}\left[e_{k}^{p+3}\right]
$$

and the proof is finished.

From Theorem 2 and by adding a new step to method M6, it is clear that the resulting scheme, denoted by M8, has order of convergence eight. Its iterative expression is

$$
\begin{aligned}
y^{(k)} & =x^{(k)}-\left[F^{\prime}\left(x^{(k)}\right)\right]^{-1} F\left(x^{(k)}\right), \\
z^{(k)} & =y^{(k)}-\left(\left[x^{(k)}, y^{(k)} ; F\right]^{-1}+\left[I-\left[F^{\prime}\left(x^{(k)}\right)\right]^{-1}\left[x^{(k)}, y^{(k)} ; F\right]\right]\left[F^{\prime}\left(x^{(k)}\right)\right]^{-1}\right) F\left(y^{(k)}\right), \\
w^{(k)} & =z^{(k)}-\left(\left[x^{(k)}, y^{(k)} ; F\right]^{-1}+\left[I-\left[F^{\prime}\left(x^{(k)}\right)\right]^{-1}\left[x^{(k)}, y^{(k)} ; F\right]\right]\left[F^{\prime}\left(x^{(k)}\right)\right]^{-1}\right) F\left(w^{(k)}\right), \\
x^{(k+1)} & =w^{(k)}-\left(\left[x^{(k)}, y^{(k)} ; F\right]^{-1}+\left[I-\left[F^{\prime}\left(x^{(k)}\right)\right]^{-1}\left[x^{(k)}, y^{(k)} ; F\right]\right]\left[F^{\prime}\left(x^{(k)}\right)\right]^{-1}\right) F\left(w^{(k)}\right),
\end{aligned}
$$

and it will be used in the numerical section.

\section{Numerical results}

To illustrate the convergence behavior of the proposed methods M6 described in (9), we consider some numerical examples and compare its performance with the one of the known schemes appeared along the manuscript and denoted by SA and WZQT. Later on, the performance of method M8 (see 11) is checked by solving a nonlinear system coming from the discretization of a partial differential equation describing a heat conduction problem.

All computations are performed in the programming package Matlab $R 2013 b$ using variable precision arithmetics with 2000 digits of mantissa. Moreover, the divided differences performed are of first order. For every method, we analyze the number of iterations (iter) needed to converge to the solution such that $\left\|x^{(k+1)}-x^{(k)}\right\|<10^{-500}$ and $\left\|F\left(x^{(k+1)}\right)\right\|<10^{-500}$ are satisfied, where $\|\cdot\|$ denotes the Euclidean norm. To verify the theoretical order of convergence $p$, we calculate the approximated computational order of convergence (ACOC) introduced in [14] as

$$
p \approx A C O C=\frac{\ln \left(\left\|x^{(k+1)}-x^{(k)}\right\| /\left\|x^{(k)}-x^{(k-1)}\right\|\right)}{\ln \left(\left\|x^{(k)}-x^{(k-1)}\right\| /\left\|x^{(k-1)}-x^{(k-2)}\right\|\right)} .
$$

Let us remark that, if the entries of vector ACOC do not stabilize their values along the iterative process, it is marked as '-'.

In Table 1, we show the numerical results obtained applying the described methods on the following academic nonlinear systems: 
- The first nonlinear system to be solved is defined by

$$
F_{1}\left(x_{1}, x_{2}, x_{3}\right)=\left(\cos x_{2}-\sin x_{1}, x_{3}^{x_{1}}-\frac{1}{x_{2}}, e^{x_{1}}-x_{3}^{2}\right)
$$

which solution is $\xi \approx(0.909569,0.661227,1.575834)$.

$-F_{2}\left(x_{1}, x_{2}, \ldots, x_{n}\right)$ is defined by the coordinate functions $f_{i}=x_{i}^{2} x_{i+1}-1$, for $i=1,2, \ldots, n-1$ and $f_{n}=x_{n}^{2} x_{1}-1$. Moreover, the searched root is $\xi \approx(1,1, \ldots, 1)^{T}$. The numerical results from this example given in Table 1 correspond to $n=50$.

- The third nonlinear system has size $4 \times 4$, being the involved function $F_{3}\left(x_{1}, x_{2}, \ldots, x_{n}\right)$, whose coordinate functions are defined by $f_{i}=x_{i}-\cos \left(2 x_{i}-\sum_{i=1}^{4} x_{i}\right), i=1,2,3,4$, being in this case $\xi \approx(0.5149,0.5149, \ldots, 0.5149)^{T}$.

- The last nonlinear system is defined by

$$
\left.F_{1}\left(x_{1}, x_{2}, x_{3}, x_{4}\right)=\left(x_{2} x_{3}+x_{4}\left(x_{2}+x_{3}\right), x_{1} x_{3}+x_{4}\left(x_{1}+x_{3}\right), x_{1} x_{2}+x_{4}\left(x_{1}+x_{2}\right), x_{1} x_{2}+x_{1} x_{3}+x_{2} x_{3}-1\right)\right),
$$

\begin{tabular}{|c|c|c|c|c|c|}
\hline$F(x), x^{(0)}$ & Methods & $\left\|x^{(k+1)}-x^{(k)}\right\|$ & $\left\|F\left(x^{(k+1)}\right)\right\|$ & iter & $A C O C$ \\
\hline \multirow{3}{*}{$\begin{array}{c}F_{1}, \\
x^{(0)}=(1.5,1.5,1.5)\end{array}$} & $\overline{\mathrm{SA}}$ & $1.28 \mathrm{e}-1968$ & $1.56 \mathrm{e}-2008$ & $\overline{77}$ & 4.0960 \\
\hline & M6 & $8.34 \mathrm{e}-1307$ & $6.18 \mathrm{e}-2008$ & 7 & 4.2100 \\
\hline & WZQT & $\mathrm{nc}$ & nc & nc & nc \\
\hline \multirow[t]{3}{*}{$x^{(0)}=(2,2,2)$} & SA & nc & nc & nc & nc \\
\hline & M6 & $6.15 \mathrm{e}-691$ & $6.03 \mathrm{e}-2008$ & 8 & 4.2939 \\
\hline & WZQT & nc & nc & nc & nc \\
\hline \multirow{3}{*}{$\begin{array}{c}F_{2}, \\
x^{(0)}=(1.5, \ldots, 1.5)\end{array}$} & $\mathrm{SA}$ & $1.14 \mathrm{e}-2076$ & 0 & 6 & 5.9935 \\
\hline & M6 & $2.13 \mathrm{e}-2234$ & 0 & 6 & 5.9955 \\
\hline & WZQT & 0 & 0 & 6 & - \\
\hline \multirow[t]{3}{*}{$x^{(0)}=(2, \ldots, 2)$} & $\mathrm{SA}$ & $1.39 \mathrm{e}-1011$ & 0 & 6 & 6.0000 \\
\hline & M6 & $7.58 \mathrm{e}-1115$ & 0 & 6 & 6.0000 \\
\hline & WZQT & nc & 0 & 7 & 7.0025 \\
\hline \multirow{3}{*}{$\begin{array}{c}F_{3} \\
x^{(0)}=(0.75, \ldots, 0.75 .5)\end{array}$} & SA & $6.28 \mathrm{e}-1107$ & 0 & 5 & 6.0000 \\
\hline & M6 & $8.32 \mathrm{e}-1118$ & 0 & 5 & 6.0000 \\
\hline & WZQT & $3.25 \mathrm{e}-1222$ & 0 & 5 & 6.9998 \\
\hline \multirow[t]{3}{*}{$x^{(0)}=(1.5, \ldots, 1.5)$} & SA & $3.84 \mathrm{e}-1267$ & 0 & 8 & 6.0000 \\
\hline & M6 & $4.81 \mathrm{e}-1619$ & 0 & 9 & 6.0000 \\
\hline & WZQT & 0 & 0 & 11 & - \\
\hline \multirow{6}{*}{$\begin{array}{c}F_{3}, \\
x^{(0)}=(-1,-1,-1,0)\end{array}$} & SA & $1.72 \mathrm{e}-1747$ & $2.7 \mathrm{e}-2008$ & 6 & 5.9651 \\
\hline & M6 & $5.71 \mathrm{e}-1847$ & $1.35 \mathrm{e}-2008$ & 6 & 5.9792 \\
\hline & WZQT & nc & nc & nc & nc \\
\hline & $\mathrm{SA}$ & $2.85 e-636$ & $2.70 \mathrm{e}-2008$ & 5 & 5.9849 \\
\hline & M6 & $2.74 \mathrm{e}-667$ & $2.70 \mathrm{e}-2008$ & 5 & 5.9425 \\
\hline & WZQT & $\mathrm{nc}$ & $\mathrm{nc}$ & nc & nc \\
\hline
\end{tabular}

being its solution $\xi \approx(-0.57735,-0.57735,-0.57735,0.288675)$.

Table 1: Numerical results for academic examples

In Table 1 we observe that the proposed sixth-order method performs equal or better than known method from Sharma and Arora, denoted by SA and it usually converges when seventh-order method WZQT does not. The ACOC of all methods confirm the theoretical result except in the first function, that shows to make the methods unstable.

\subsection{D Heat conduction equation}

In this section we consider a particular case of one-dimensional nonlinear partial differential equation

$$
u_{x} x=u_{t}+\phi\left(x, t, u, u_{x}\right), \quad x \in[a, b], t \geq 0,
$$

with initial condition $u(x, 0)=g(x), x \in[a, b]$ and boundary conditions $u(a, t)=C_{1}(t), u(b, t)=C_{2}(t), t \geq 0$.

Problems of this type arise in numerous applications from science and engineering, such as fluid flow at high Reynolds number, heat transfer with small diffusion parameters, filtration of liquids, gas dynamics, heat conduction, elasticity, chemical reaction, etc. 
In our study a particular case is used, corresponding to the following heat conduction equation

$$
u_{x x}=u_{t}+u_{x}-u^{2}+f(x, t), \quad 0 \leq x \leq 1, \quad t \geq 0,
$$

where $f(x, t)=e^{-t}\left(-\pi \cos (\pi x)-\left(\pi^{2}-2\right) \sin (\pi x)\right)$. The initial condition is $u(x, 0)=\sin (\pi x)$ and the boundary conditions are

$$
u(0, t)=0, \quad u(1, t)=0 .
$$

By applying an implicit method of finite differences we can transform problem (12) in a family of nonlinear systems, which provides the approximated solution in a time $t_{k}$ from the approximated solution in $t_{k-1}$. We choose the spacial step $h=1 / n x$ and the temporal step $k=T_{\max } / n t$, where $n x$ and $n t$ are the number of $x$-subintervals and $t$-subintervals, respectively, and $T_{\max }$ the final instant of our study. So, we have selected a grid of domain $[0,1] \times\left[0, T_{\max }\right]$ with points $\left(x_{i}, t_{j}\right)$,

$$
x_{i}=0+i h, \quad i=0,1, \ldots, n x, \quad t_{j}=0+j k, \quad j=0,1, \ldots, n t .
$$

We want to estimate the solution of (12) at these points, by transforming it in many nonlinear systems, as much as the number of $t_{j}$. To do that, we use the following approximations:

$$
\begin{aligned}
u_{x}(x, t) & \approx \frac{u(x+h, t)-u(x-h, t)}{2 h}, \\
u_{t}(x, t) & \approx \frac{u(x, t)-u(x, t-k)}{k}, \\
u_{x x}(x, t) & \approx \frac{u(x+h, t)-2 u(x, t)+u(x-h, t)}{h^{2}} .
\end{aligned}
$$

Denoting by $u_{i, j}$ the estimation of the solution at $\left(x_{i}, t_{j}\right)$ and by replacing them in (12), we construct the following nonlinear system

$$
\frac{u_{i+1, j}-2 u_{i, j}+u_{i-1, j}}{h^{2}}=\frac{u_{i, j}-u_{i, j-1}}{k}+\frac{u_{i+1, j}-u_{i-1, j}}{2 h}-u_{i, j}^{2}+f\left(x_{i}, t_{j}\right),
$$

for $i=1,2, \ldots, n x-1$ and $t=1,2, \ldots, n t$. Some algebraic manipulations allow us to transform this system in:

$$
(2 k-k h) u_{i+1, j}+\left(-4 k-2 h^{2}\right) u_{i, j}+(2 k+k h) u_{i-1, j}+2 k h^{2} u_{i, j}^{2}=2 k h^{2} f\left(x_{i}, t_{j}\right)-2 h^{2} u_{i, j-1},
$$

for $i=1,2, \ldots, n x-1$ and $j=1,2, \ldots, n t$. For a fixed $j$, we have the following nonlinear system of size $(n x-1) \times$ $(n x-1)$ :

$$
\begin{aligned}
& (2 k-k h) u_{2, j}-\left(4 k+2 h^{2}\right) u_{1, j}+2 k h^{2} u_{1, j}^{2}=2 k h^{2} f\left(x_{1}, t_{j}\right)-2 h^{2} u_{1, j-1} \\
& (2 k-k h) u_{i+1, j}-\left(4 k+2 h^{2}\right) u_{i, j}+(2 k+k h) u_{i-1, j}+2 k h^{2} u_{i, j}^{2}=2 k h^{2} f\left(x_{i}, t_{j}\right)-2 h^{2} u_{i, j-1}, i=2,3, \ldots, n x-2, \\
& -\left(4 k+2 h^{2}\right) u_{n x-1, j}+(2 k+k h) u_{n x-2, j}+2 k h^{2} u_{n x-1, j}^{2}=2 k h^{2} f\left(x_{n x-1}, t_{j}\right)-2 h^{2} u_{n x-1, j-1} .
\end{aligned}
$$

The unknowns of this system are $u_{1, j}, u_{2, j}, \ldots, u_{n x-1, j}$, that is, the approximations of the solution in $t_{j}$. We observe that for solving this system we need the solution in $t_{j-1}$.

We are going to solve this system for some values of $T_{\max }$, using in each case different values of $n t, n x=20$ and method M8. As initial guess we use the approximated solution of the previous $t_{j}$. We compare the obtained values with the exact solution $u(x, t)=e^{-t} \sin (\pi x)$ and a maximum number of iterations 4000, in order to analyze the stability and consistence of the new method. We also calculate the mean number of iterations needed for solving all the nonlinear systems needed for completing the process.

All computations are performed using variable precision arithmetics with 500 digits of mantissa. For every value of $T_{\max }$, we analyze the number of iterations (iter) needed to converge to the solution such that $\left\|F\left(x^{(k+1)}\right)\right\|<$ $10^{-12}$ is satisfied, where $\|\cdot\|$ denotes the Euclidean norm. The number of spatial subinterval is $n x=20$ in all cases.

As it was expected, in Table 2 is observed that, we must add more temporal subintervals when $T_{\max }$ is increased. Moreover, due to the high order of convergence of M8, the mean number of required iterations is very small, as well as the value of $\left\|F\left(x^{(k+1)}\right)\right\|$ respect the exact error. The exact error is much higher than $\left\|F\left(x^{(k+1)}\right)\right\|$ due to the order of the divided difference used in the discretization process, $O\left(h^{2}+k\right)$.

In Figure 1a we show the approximated solution of the problem when $T_{\max }=0.3$, by taking $n t=500$ and $n x=20$. It is a good approximation if we compare it with the exact solution (Figure 1b). In Figure 2 the absolute value of the exact error is presented. 


\begin{tabular}{|c|c|c|c|c|c|}
\hline$T_{\max }$ & $n t$ & Exact error & $\left\|F\left(x^{(k+1)}\right)\right\|$ & iter & CPU-Time \\
\hline \hline 0.1 & 100 & 0.0073 & $9.746 \mathrm{e}-14$ & 1 & 155.07 \\
0.1 & 500 & 0.0073 & $9.82 \mathrm{e}-17$ & 1 & 759.13 \\
\hline \hline 0.3 & 100 & $\mathrm{nc}$ & $\mathrm{nc}$ & $\mathrm{nc}$ & $\mathrm{nc}$ \\
0.3 & 500 & 0.0186 & $4.94 \mathrm{e}-15$ & 1 & 725.72 \\
0.3 & 1000 & 0.0186 & $2.63 \mathrm{e}-16$ & 1 & 1478.72 \\
\hline \hline 0.5 & 100 & $\mathrm{nc}$ & $\mathrm{nc}$ & $\mathrm{nc}$ & $\mathrm{nc}$ \\
0.5 & 500 & 0.0247 & $3.39 \mathrm{e}-14$ & 1 & 757.55 \\
0.5 & 1000 & 0.0247 & $1.17 \mathrm{e}-15$ & 1 & 1473.18 \\
\hline \hline 0.7 & 100 & $\mathrm{nc}$ & $\mathrm{nc}$ & $\mathrm{nc}$ & $\mathrm{nc}$ \\
0.7 & 500 & 0.0263 & $1.29 \mathrm{e}-13$ & 1.0160 & 786.98 \\
0.7 & 1000 & 0.0263 & $3.19 \mathrm{e}-15$ & 1 & 1589.17 \\
\hline \hline 1 & 100 & $\mathrm{nc}$ & $\mathrm{nc}$ & $\mathrm{nc}$ & $\mathrm{nc}$ \\
1 & 500 & 0.0245 & $4.71 \mathrm{e}-13$ & 1.9420 & 1407.79 \\
1 & 1000 & 0.0246 & $1.003 \mathrm{e}-14$ & 1 & 1508.01 \\
\hline \hline 1.5 & 500 & $\mathrm{nc}$ & $\mathrm{nc}$ & $\mathrm{nc}$ & $\mathrm{nc}$ \\
1.5 & 1000 & 0.0181 & $3.23 \mathrm{e}-14$ & 1.0310 & 1564.07 \\
1.5 & 1500 & 0.0181 & $3.30 \mathrm{e}-15$ & 1 & 2393.18 \\
\hline \hline 2 & 1000 & 0.0121 & $5.68 \mathrm{e}-14$ & 1.4710 & 2328.18 \\
2 & 1500 & 0.0121 & $5.84 \mathrm{e}-15$ & 1 & 2424.78 \\
\hline \hline
\end{tabular}

Table 2: Numerical results for different values of $T_{\max }$

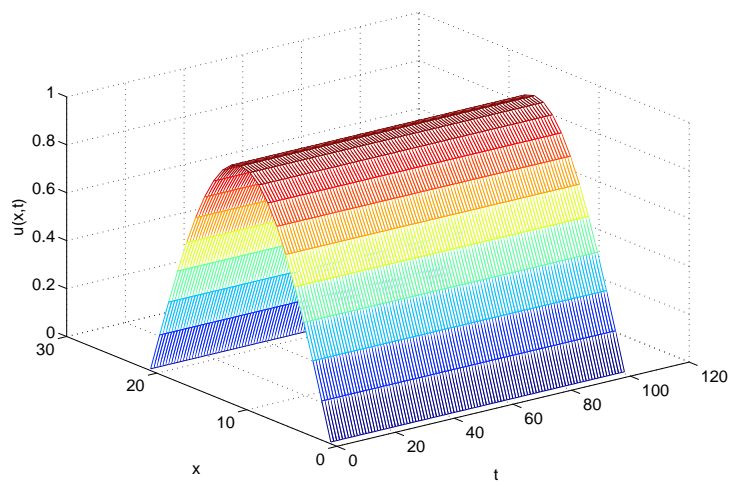

(a) Approximated

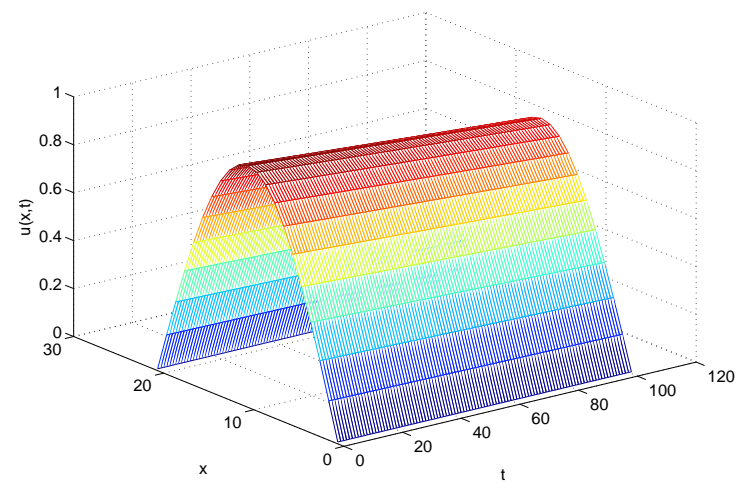

(b) Exact

Fig. 1: Graphics of the approximated and exact solutions for $t \in[0,0.3]$

\section{Conclusions}

By summarizing, we have defined a class of biparametric iterative methods for solving systems of nonlinear equations of arbitrary even order. This family holds known methods as Sharma et al. 6th-scheme and also other methods as stable as the known ones. From the numerical point of view, two simple elements of order 6 and 8 have been selected and tested numerically among other schemes of same and higher order of convergence on academical examples and also on a heat conduction problem. Its good performance has been showed, even when the other methods fail.

\section{Acknowledgements}

This research was partially supported by Ministerio de Economía y Competitividad MTM2014-52016-C02-2-P and FONDOCYT 2014-1C1-088 República Dominicana. 


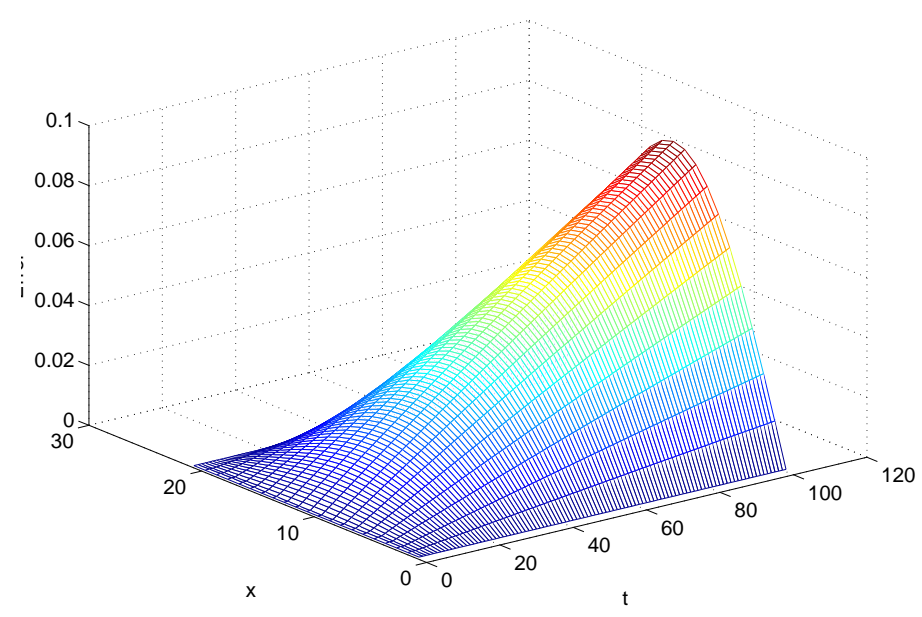

Fig. 2: Graphic of the exact error for $t \in[0,0.3]$

\section{References}

1. R. Escobedo, L.L. Bonilla, Numerical methods for quantum drift-diffusion equation in semiconductor Phisics, Math. Chem. 40(1) 3-13 (2006)

2. S. J. Preece, J.Villingham, A.C. King, Chemical clock reactions: The effect of precursor consumtion, Math. Chem. 26 47-73 (1999)

3. H. Montazeri, F. Soleymani, S. Shateyi, S. S. Motsa, On a new method for computing the numerical solution of systems of nonlinear equations, J. Appl. Math. 2012 ID. 751975, 15 pages (2012)

4. J. L. Hueso, E. Martínez, C. Teruel, Convergence, effiency and dinamimics of new fourth and sixth order families of iterative methods for nonlinear systems, J. Comput. Appl. Math. 275 412-420 (2015)

5. J. R. Sharma, H. Arora, Efficient Jarratt-like methods for solving systems of nonlinear equations, Calcolo 51 $193-210$ (2014)

6. X. Wang, T. Zhang, W. Qian, M. Teng, Seventh-order derivative-free iterative method for solving nonlinear systems, Numer. Algor. 70 545-558 (2015)

7. J. R. Sharma, H. Arora, On efficient weighted-Newton methods for solving systems of nonlinear equations, Appl. Math. Comput. 222 497-506 (2013)

8. A. Cordero, J. G. Maimó, J. R. Torregrosa, M. P. Vassileva, Solving nonlinear problems by Ostrowski-Chun type parametric families, J. Math. Chem. 53 430-449 (2015)

9. A.M. Ostrowski, Solution of equations and systems of equations. Prentice-Hall, Englewood Cliffs, New York (1964)

10. C. Chun, Construction of Newton-like iterative methods for solving nonlinear equations, Numerical Mathematics 104 297-315 (2006)

11. A. Cordero, J.L. Hueso, E. Martínez, J.R. Torregrosa, A modified Newton-Jarratt's composition, Numer. Algor. 55 87-99 (2010)

12. J.M. Ortega, W.C. Rheinboldt, Iterative Solution of Nonlinear Equations in Several Variables. Academic, New York (1970)

13. C. Hermite, Sur la formule dinterpolation de Lagrange, Reine Angew. Math. 84 70-79 (1878)

14. A. Cordero, J.R. Torregrosa, Variants of Newton's method using fifth-order quadrature formulas, Appl. Math. Comput. 190 686-698 (2007) 